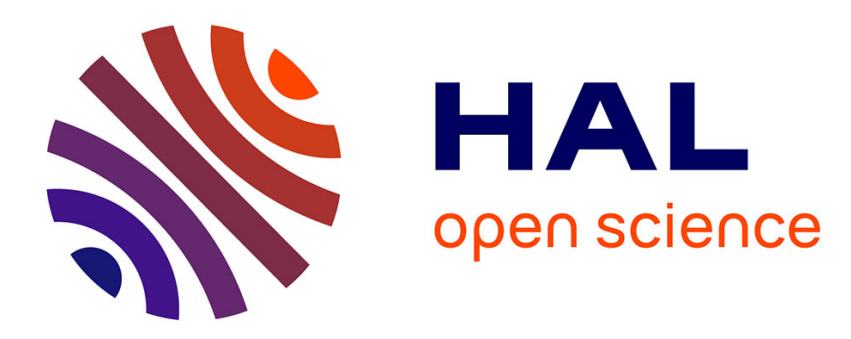

\title{
On charge conservation in a gravitational field
}

\author{
Mayeul Arminjon
}

\section{To cite this version:}

Mayeul Arminjon. On charge conservation in a gravitational field. Nineteenth International Conference on Geometry, Integrability and Quantization, Ivaïlo M. Mladenov, Jun 2017, Varna, Bulgaria. hal-01613187

\section{HAL Id: hal-01613187 \\ https://hal.science/hal-01613187}

Submitted on 12 Oct 2017

HAL is a multi-disciplinary open access archive for the deposit and dissemination of scientific research documents, whether they are published or not. The documents may come from teaching and research institutions in France or abroad, or from public or private research centers.
L'archive ouverte pluridisciplinaire HAL, est destinée au dépôt et à la diffusion de documents scientifiques de niveau recherche, publiés ou non, émanant des établissements d'enseignement et de recherche français ou étrangers, des laboratoires publics ou privés. 


\title{
On charge conservation in a gravitational field
}

\author{
Mayeul Arminjon \\ Univ. Grenoble Alpes, CNRS, Grenoble INP, 3SR, F-38000 Grenoble, France
}

\begin{abstract}
According to the "gravitationally-modified" Maxwell equations that were proposed for an alternative scalar theory with an "ether", electric charge would not be conserved in a time-dependent gravitational field. We define an asymptotic expansion scheme for the electromagnetic field in a weak gravitational field. This allows us to assess the amounts of charge production or destruction which are thus predicted. These amounts seem high enough to discard that version of the gravitationally-modified Maxwell equations. We show that this failure is due to the former assumption of additivity of the energy tensors: an "interaction energy tensor" has to be added. Then the standard Maxwell equations in a curved spacetime become compatible with that scalar theory, and they predict charge conservation.
\end{abstract}

\section{Introduction}

An alternative theory of gravitation based on a scalar field alone, and having a preferred reference frame, has been proposed previously [1, 2, 17. Recently, equations for the electromagnetic field in the presence of a gravitational field have been derived for this theory [9] and it has been found that they lead to a violation of the conservation of electric charge. The aim of this contribution is to give a short account of the work that has been done to assess the magnitude of this effect, and to outline the consequences that followed for the theory from this assessment. (A detailed account has been given elsewhere [10.) Let us first summarize the three main motivations for this "scalar ether theory" of gravitation, in short SET. 
1) The first motivation is to extend to the situation with gravitation the Lorentz-Poincaré version of special relativity. The latter version is more often called "Lorentz ether theory", although undoubtedly Poincaré made essential contributions to it. This is the theory according to which the "ether" is a rigid inertial frame such that: (i) any object that is moving with respect to it is subjected to the Lorentz contraction, and (ii) in this frame, energy propagation is isotropic, in particular the Maxwell equations are assumed valid in that frame. This theory obtains the Lorentz transformation and then all "relativistic" effects as following from (a) "absolute" effects of motion through that ether, and (b) Clock synchronization. It is exposed in particular in Prokhovnik [19], and a summary can be found in Ref. [2]. In that theory, the restriction " $v<c$ " is not absolute, it concerns mass particles.

2) Newton's $1 / r^{2}$ force, which acts instantaneously at a distance, cannot be intuitively understood. (This was already Newton himself's opinion.) Einstein's general relativity (GR) sees gravity as propagating at the velocity of light $c$, but says that ("free") particles follow geodesic curves in the fourdimensional "space-time" manifold V endowed with a curved Lorentzian metric $\boldsymbol{\gamma}$. This gives a fundamental physical status to the spacetime - although, to more than one physicist, spacetime looks more like a mere mathematical construction. (The latter certainly is its status in the Lorentz-Poincaré version of special relativity.) In contrast, SET makes gravity thinkable as the pressure force of the ether: Archimedes' thrust on extended particles seen as organized flows in the ether [1].

3) Despite its successes, GR has problems: (i) The unavoidable singularities (e.g. in a gravitational collapse and in the "big bang"). (ii) The interpretation of the necessary gauge condition. (iii) The problems regarding the coupling with quantum theory. (iv) The need for dark matter. (v) The need for dark energy. In contrast: (i) SET has no singularity either in a gravitational collapse [4] or in the past high-density state [5] and (ii) no gauge condition. (iii) The assumed preferred reference frame makes it possible to write quantum theory in a gravitational field unambiguously. For instance, this avoids the non-uniqueness problem of the covariant Dirac theory [8]. (iv) Preferred-frame effects should be more important at large scales (for they have more time to accumulate) and might possibly contribute to explain motion at a galactical scale. (v) That theory necessarily predicts accelerated expansion, without assuming any dark energy [5]. 


\section{Equations for the Electromagnetic Field}

SET has a preferred reference frame $\mathcal{E}$. It has also a curved spacetime metric noted $\gamma$. The equations below are usually valid only in coordinates adapted to that reference frame and such that the synchronization condition $\gamma_{0 i}=$ $0(i=1,2,3)$ is verified. The spatial metric in the frame $\mathcal{E}$ [14, 17, 11, 3] will be noted $\boldsymbol{g}$. The first Maxwell group is unchanged. In terms of the antisymmetric field tensor $\boldsymbol{F}$ :

$$
F_{\sigma \mu, \nu}+F_{\mu \nu, \sigma}+F_{\nu \sigma, \mu}=F_{\sigma \mu ; \nu}+F_{\mu \nu ; \sigma}+F_{\nu \sigma ; \mu}=0 .
$$

(Semicolon means a covariant derivative associated with the metric $\gamma$. The first equality is an identity for an antisymmetric tensor field and a torsionless connection, as is the metric (Levi-Civita) connection.) To get the second group [9], we used the equation for continuum dynamics that is valid in SET and we applied it to the charged medium subjected to the Lorentz force, assuming that:

(i) The total energy(-momentum) tensor is $\boldsymbol{T}_{\text {total }}=\boldsymbol{T}_{\text {charged medium }}+\boldsymbol{T}_{\text {field }}$.

(ii) The total energy tensor $\boldsymbol{T}_{\text {total }}$ obeys the general equation for continuum dynamics, without any non-gravitational force.

After some algebra using the explicit form of the energy tensor $\boldsymbol{T}_{\text {field }}$ of the electromagnetic (e.m.) field, this gives (writing the equations "in SI units"):

$$
F_{\sigma}^{\mu} F_{; \nu}^{\sigma \nu}=\mu_{0}\left[b^{\mu}\left(\boldsymbol{T}_{\text {field }}\right)-F_{\sigma}^{\mu} J^{\sigma}\right]
$$

where

$$
b^{0}(\boldsymbol{T}) \equiv \frac{1}{2} \gamma^{00} g_{i j, 0} T^{i j}, \quad b^{i}(\boldsymbol{T}) \equiv \frac{1}{2} g^{i j} g_{j k, 0} T^{0 k} .
$$

\section{Charge Non-Conservation}

If $\operatorname{det} \boldsymbol{F} \neq 0$ (where $\boldsymbol{F}$ is the matrix $\boldsymbol{F} \equiv\left(F^{\mu}{ }_{\nu}\right)$ ), which is equivalent [9] to $\mathbf{E . B} \neq 0$ with $\mathbf{E}$ and $\mathbf{B}$ the electric and magnetic fields, we get from Eq. (2) [9]:

$$
\hat{\rho} \equiv J_{; \mu}^{\mu}=\left(G^{\mu}{ }_{\nu} b^{\nu}\left(\boldsymbol{T}_{\text {field }}\right)\right)_{; \mu}, \quad\left(G^{\mu}{ }_{\nu}\right) \equiv\left(F^{\mu}{ }_{\nu}\right)^{-1} .
$$


Thus, charge conservation $\left(J_{; \mu}^{\mu}=0\right)$ is not true in general with the second group (2), in contrast with what happens with the second Maxwell group assumed in GR and other metric theories of gravitation [9].

Let $\Omega$ be any "substantial" domain of the charged continuum, i.e., it is followed in its motion. We can prove that the evolution rate of the charge contained in $\Omega$ is

$$
\frac{\mathrm{d}}{\mathrm{d} t}\left(\int_{\Omega} \delta q\right)=\int_{\Omega} \hat{\rho} \sqrt{-\gamma} \mathrm{d}^{3} x \quad\left(\gamma \equiv \operatorname{det}\left(\gamma_{\mu \nu}\right)\right)
$$

in any coordinates $x^{\mu}$. (Here $t \equiv x^{0} / c$.) Of course the domain $\Omega$ as well as its boundary depend on $t$ in general spatial coordinates $x^{i}$.

\section{Weak-Field Approximation of the Gravita- tional Field}

The gravitational field is assumed weak and slowly varying for the system of interest S (e.g. the Earth with some e.m. source on it). We use an asymptotic post-Newtonian $(\mathrm{PN})$ scheme. This scheme associates with $\mathrm{S}$ a family $\left(\mathrm{S}_{\lambda}\right)$ of systems, depending on $\lambda \rightarrow 0$, with $\lambda=1 / c^{2}$ in a specific $\lambda$-dependent time unit [7]. It leads to write Taylor expansions with respect to $c^{-2}$, using also a $\lambda$-dependent mass unit. E.g.

$$
\beta \equiv \sqrt{\gamma_{00}}=1-U c^{-2}+O\left(c^{-4}\right)
$$

where $U \geq 0$ is the Newtonian potential, which obeys the Poisson equation [7]. The spatial metric assumed in the theory is [7]:

$$
\boldsymbol{g}=\beta^{-2} \boldsymbol{g}^{0}
$$

with $\boldsymbol{g}^{0}$ an invariable Euclidean metric. We will take Cartesian coordinates for $\boldsymbol{g}^{0}$, i.e., $g_{i j}^{0}=\delta_{i j}$.

\section{Asymptotic Expansions for the E.M. Field and Current}

We assume that $\boldsymbol{F}$ and the 4-current $\mathbf{J}$ depend smoothly on $\lambda$, hence they too admit Taylor expansions w.r.t. $\lambda$ (with $\lambda=c^{-2}$ in the specific $\lambda$-dependent 
units):

$$
\boldsymbol{F}=c^{n}\left(\stackrel{0}{\boldsymbol{F}}+c^{-2} \stackrel{1}{\boldsymbol{F}}+O\left(c^{-4}\right)\right)
$$

and

$$
\mathbf{J}=c^{m}\left(\stackrel{0}{\mathbf{J}}+c^{-2} \stackrel{1}{\mathbf{J}}+O\left(c^{-4}\right)\right) .
$$

The integers $n$ and $m$ are not known and can be positive, negative, or zero. Since $\lambda=1 / c^{2}$ is the gravitational weak-field parameter, this means that the fields $\boldsymbol{F}$ and $\mathbf{J}$ are not assumed weak. Moreover, $\boldsymbol{F}$ is not assumed slowly varying: in this respect, the expansions (8)-(9) are similar to postMinkowskian (PM) expansions.

\section{Expansion of the Modified Maxwell Second Group}

For the PM-like expansions (8)-(9), the time variable (such that the expansions are true at a fixed value of it) is $x^{0}=c T$ [6, 12], not $T$ as it is for PN expansions [7, 12]. (This is not neutral since $c=\sqrt{\lambda^{-1}}$ with $\lambda$ the weak-field parameter.) Moreover, from dimensional analysis we get that, with the $\lambda$ dependent units, we have $\mu_{0}=\mu_{00} c^{2}$ with $\mu_{00}$ a constant. From this, we find by examining the orders in Eq. (2) that we must have

$$
2 n=n+m+2, \quad \text { i.e. } \quad m=n-2 .
$$

Using the foregoing, one gets the lowest-order term in the weak-field expansion of (2) as

$$
\stackrel{0}{F}^{\mu}{ }_{\sigma} \stackrel{0}{F}_{, \nu}^{\sigma \nu}=-\mu_{00} \stackrel{0}{F}^{\mu}{ }_{\sigma} \stackrel{0}{J}^{\sigma} .
$$

Thus if $\stackrel{0}{\boldsymbol{F}} \equiv\left(\stackrel{0}{F}_{\nu}^{\sigma}\right)$ is invertible, it is an exact solution of the flat-spacetime Maxwell equation:

$$
\stackrel{0}{F}^{\sigma \nu}{ }_{, \nu}=-\mu_{00} \stackrel{0}{J}^{\sigma} .
$$

\section{Expansion of the Charge Production Rate}

Due to 8 - 8 - $99, \stackrel{0}{\boldsymbol{F}}, \stackrel{0}{\boldsymbol{G}}$, etc., do not have the physical dimensions of the corresponding fields $\boldsymbol{F}, \boldsymbol{G}$, etc. However, $\boldsymbol{F}_{1} \equiv c^{n} \boldsymbol{F} \quad$ and $\quad \mathbf{J}_{1} \equiv c^{m} \mathbf{J}^{0}$ are solu- 
tions of the flat-spacetime Maxwell equation with the correct dimensions in the SI units. Define $\boldsymbol{T}_{1}$ as the e.m. T-tensor associated with $\boldsymbol{F}_{1}$. Assume matrix $\boldsymbol{F}_{1}$, with components $\left(F_{1}\right)^{\sigma}{ }_{\nu}$, is invertible. Define $\boldsymbol{G}_{1} \equiv \boldsymbol{F}_{1}{ }^{-1}$. Using (8), we can show that

$$
\hat{\rho}=c^{-3}\left[\left(G_{1}^{\mu 0} T_{1}^{j j}-G_{1}^{\mu i} T_{1}^{0 i}\right) \partial_{T} U\right]_{, \mu}+O\left(c^{-5}\right) .
$$

Expressing $\boldsymbol{F}$ in terms of the electric and magnetic fields $\mathbf{E}$ and $\mathbf{B}$, we can rewrite 13 as

$$
\hat{\rho}=c^{-3}\left(e^{i} \partial_{T} U\right)_{, i}+O\left(c^{-5}\right)
$$

with

$$
e^{i}=\left(\begin{array}{c}
\frac{B_{1}{ }^{3} c^{2}+B_{1} B_{2}{ }^{2} c^{2}+B_{1} B_{3}{ }^{2} c^{2}+B_{1} E_{1}{ }^{2}-B_{1} E_{2}{ }^{2}-B_{1} E_{3}{ }^{2}+2 B_{2} E_{1} E_{2}+2 B_{3} E_{1} E_{3}}{2 c \mu_{0}\left(B_{1} E_{1}+B_{2} E_{2}+B_{3} E_{3}\right)} \\
\frac{B_{1}{ }^{2} B_{2} c^{2}+2 B_{1} E_{1} E_{2}+B_{2}{ }^{3} c^{2}+B_{2} B_{3}{ }^{2} c^{2}-B_{2} E_{1}{ }^{2}+B_{2} E_{2}{ }^{2}-B_{2} E_{3}{ }^{2}+2 B_{3} E_{2} E_{3}}{2 c \mu_{0}\left(B_{1} E_{1}+B_{2} E_{2}+B_{3} E_{3}\right)} \\
\frac{B_{1}{ }^{2} B_{3} c^{2}+2 B_{1} E_{1} E_{3}+B_{2}^{2} B_{3} c^{2}+2 B_{2} E_{2} E_{3}+B_{3}{ }^{3} c^{2}-B_{3} E_{1}{ }^{2}-B_{3} E_{2}{ }^{2}+B_{3} E_{3}{ }^{2}}{2 c \mu_{0}\left(B_{1} E_{1}+B_{2} E_{2}+B_{3} E_{3}\right)}
\end{array}\right) .
$$

To evaluate (14), we have to assess $\partial_{T} U$ and $\partial_{T} \nabla U$. Both have to be calculated in the preferred reference frame $\mathcal{E}$, since it is in that frame that the equations are valid. The system of interest producing the e.m. field should move through $\mathcal{E}$, with a velocity field $\mathbf{v}$. One expects that $|\mathbf{v}|$ should be in the range $10-1000 \mathrm{~km} / \mathrm{s}$. We can show that, for such velocities, the main contribution to $\partial_{T} U$ comes from the translation motion of a nearly spherically symmetric body (with center $\mathbf{a}(T)$ ) through $\mathcal{E}$ :

$$
\partial_{T} U \simeq-\mathbf{V} \cdot \nabla U \simeq \frac{G M(r)}{r^{2}} \mathbf{V} \cdot \mathbf{e}_{r}, \quad r \equiv|\mathbf{x}-\mathbf{a}(T)|, \quad \mathbf{e}_{r} \equiv(\mathbf{x}-\mathbf{a}(T)) / r
$$

with $\mathbf{V} \equiv \dot{\mathbf{a}}$ and $M(r) \equiv 4 \pi \int_{0}^{r} u^{2} \rho(u) \mathrm{d} u, \quad \rho(r)$ being the Newtonian mass density. On the Earth's surface, this gives $\partial_{T} U \simeq g V_{r} \lesssim 10 \mathrm{~V} \simeq 10^{5}$ (MKSA) for $V=10 \mathrm{~km} / \mathrm{s}$. If moreover the rotating spherical body is homogeneous (which can be assumed to get an order-of-magnitude estimate), we have

$$
\partial_{T} \nabla U=\frac{G M(r)}{r^{3}} \mathbf{V}
$$

On Earth: $\partial_{T} \nabla U \simeq g \mathbf{V} / R$, hence $\left|\partial_{T} \nabla U\right| \simeq 10^{-2}(\mathrm{MKSA})$ for $V=10 \mathrm{~km} / \mathrm{s}$. 


\section{Application to Representative Fields}

\subsection{Case of a Plane Wave}

A monochromatic, "pure", plane e.m. wave propagating in the direction i $\| O x$ has

$$
E^{1}=0, E^{i}=E_{0}^{i} \cos \left(k x-\omega T+\varphi_{i}\right)(i=2,3), \quad c \mathbf{B}=\mathbf{i} \wedge \mathbf{E} .
$$

Then of course $\mathbf{E} . \mathbf{B}=0$, hence the field matrix $\boldsymbol{F} \equiv\left(F^{\mu}{ }_{\nu}\right)$ is not invertible. But we may add any constant e.m. field $\left(\mathbf{E}^{\prime}, \mathbf{B}^{\prime}\right)$ : the field is still a plane wave (though not a pure one, in that the condition $c \mathbf{B}=\mathbf{i} \wedge \mathbf{E}$ is lost). Then generically $\boldsymbol{F}$ is invertible. Moreover, $e^{i}$ [Eq. (14)] has $e_{, i}^{i}=0$, for $e^{1}=0$ and $e^{i}=e^{i}\left(x^{1}\right)$. Neglecting the term $c^{-3} e^{i}\left(\partial_{T} \widetilde{U}\right)_{, i}$ in view of (17) and the extremely small figure $c^{-3} \simeq 3.7 \times 10^{-26}$ (MKSA), we get that

$$
\hat{\rho}=0 \quad \text { (Plane wave, } c^{-3} e^{i}\left(\partial_{T} U\right)_{, i} \text { neglected). }
$$

However, for very strong values of the constant e.m. field, the neglected term may give high values of $\hat{\rho}$. (Check the case without the wave part.)

\subsection{The Case with Hertzian Dipoles}

Hertz's oscillating dipole is the charge distribution

$$
\rho=T_{\mathbf{d}, \mathbf{b}, \omega} \equiv-e^{-\mathrm{i} \omega t} \mathbf{d} . \nabla \delta_{\mathbf{b}}
$$

with $\mathbf{b}$ the dipole position, and $\mathbf{d}$ the dipole vector. The associated 3 -current is:

$$
\mathbf{j}=-\mathrm{i} \omega \mathbf{d} e^{-\mathrm{i} \omega t} \delta_{\mathbf{b}}
$$

With this source distribution, corresponds a solution [13, 16] of the flat Maxwell equations (in the distributional sense):

$$
\begin{gathered}
\mathbf{E}=\alpha\left\{\frac{k^{2}}{r}(\mathbf{d}-(\mathbf{n} . \mathbf{d}) \mathbf{n}) \cos \varphi+[3(\mathbf{n} . \mathbf{d}) \mathbf{n}-\mathbf{d}]\left(\frac{\cos \varphi}{r^{3}}+\frac{k \sin \varphi}{r^{2}}\right)\right\} \\
\mathbf{B}=\beta k^{2}(\mathbf{n} \wedge \mathbf{d})\left(\frac{\cos \varphi}{r}-\frac{\sin \varphi}{k r^{2}}\right), \quad k=\frac{\omega}{c}, \quad \varphi \equiv k r-\omega t .
\end{gathered}
$$

Here $\alpha \equiv \frac{1}{4 \pi \epsilon_{0}}=9 \times 10^{9}, \quad \beta \equiv \frac{c}{4 \pi} \simeq 2.39 \times 10^{7}$ (MKSA). This solution can be shown to be exact. It has $\mathbf{E} . \mathbf{B}=0$. However, adding dipoles with different 
b's and d's gives generically $\mathbf{E} . \mathbf{B} \neq 0$.

We thus consider a group of Hertzian dipoles. All of them are at rest in a common frame $\mathcal{E}_{\mathbf{V}}$, moving at a constant velocity $\mathbf{V}$ w.r.t. $\mathcal{E}$. Their e.m. field is obtained in the moving frame $\mathcal{E}_{\mathbf{V}}$ by summing the fields (22)-(23) of each dipole. It is then Lorentz-transformed to $\mathcal{E}$. In view of $(14)$, we compute

$$
\hat{\rho}(T, \mathbf{x})=c^{-3}\left(e^{i} \partial_{T} U\right)_{, i} \approx c^{-3} \int_{\partial \mathrm{C}_{\mathcal{E}}(T)} e^{i} n_{i} \partial_{T} U \mathrm{~d} S / v\left(\mathrm{C}_{\mathcal{E}}(T)\right) .
$$

Here, $\mathrm{C}$ being a small cube bound to the moving frame $\mathcal{E}_{\mathbf{V}}$, centered at the calculation point $\mathbf{x}$, we denote $\mathrm{C}_{\mathcal{E}}(T)$ the domain occupied by this cube at the time $T$ in the ether frame $\mathcal{E}$. ( $\mathbf{n}$ is the external normal to the boundary $\partial \mathrm{C}_{\mathcal{E}}(T)$ of the domain $\mathrm{C}_{\mathcal{E}}(T), \mathrm{d} S$ is the surface element on $\partial \mathrm{C}_{\mathcal{E}}(T)$, and $v\left(\mathrm{C}_{\mathcal{E}}(T)\right)$ is the volume of $\left.\mathrm{C}_{\mathcal{E}}(T).\right)$ For three dipoles with $d=100 \mathrm{nC} . \mathrm{m}$, $\nu=100 \mathrm{MHz}(\lambda=3 \mathrm{~m})$, situated at $\lesssim \lambda$ from one another, we get fields

$E \lesssim$ a few $10^{5} \mathrm{~V} / \mathrm{m}, B \lesssim 15 \mathrm{~T}$. With $V=10 \mathrm{~km} / \mathrm{s}, \hat{\rho}(T, \mathbf{x})$ has peaks at $\approx \pm 2 \times 10^{8} \mathrm{e} / \mathrm{m}^{3} /$ period. This seems untenable, even though the peaks are very narrow and their sign alternates in space.

Hence, this version of the gravitationally-modified Maxwell equations seems discarded.

\section{The Reason for the Problem and its Solu- tion}

\subsection{Why Were'nt These the Right Maxwell Equations of the Theory?}

In SET, the dynamical equation for a general continuous medium having a well-defined velocity field $\mathbf{v}$, subjected to an external force density field $\mathbf{f}$, is [9]:

$$
T_{\text {medium } ; \nu}^{0 \nu}=b^{0}\left(\boldsymbol{T}_{\text {medium }}\right)+\frac{\mathbf{f} . \mathbf{v}}{c \beta}, \quad T_{\text {medium } ; \nu}^{i \nu}=b^{i}\left(\boldsymbol{T}_{\text {medium }}\right)+f^{i} .
$$

Assumption (i) (Sect. 2) says: $\boldsymbol{T}_{\text {total }}=\boldsymbol{T}_{\text {charged medium }}+\boldsymbol{T}_{\text {field }}$. 
Assumption (ii) (Sect. 2) states: $\quad T_{\text {total } ; \nu}^{0 \nu}=b^{0}\left(\boldsymbol{T}_{\text {total }}\right), \quad T_{\text {total } ; \nu}^{i \nu}=b^{i}\left(\boldsymbol{T}_{\text {total }}\right)$.

Assumptions (i) and (ii), plus Eq. (25) with "medium" = "charged medium", lead easily to:

$$
T_{\text {field } ; \nu}^{0 \nu}=b^{0}\left(\boldsymbol{T}_{\text {field }}\right)-\frac{\mathbf{f . v}}{c \beta}, \quad T_{\text {field } ; \nu}^{i \nu}=b^{i}\left(\boldsymbol{T}_{\text {field }}\right)-f^{i} .
$$

This has the form (25) applied to the e.m. field ("medium" = "field"), with $f_{\text {field }}^{i}=-f_{\text {charged medium }}^{i} \equiv-f^{i}$ and $\mathbf{v}_{\text {field }}=\mathbf{v}_{\text {charged medium }} \equiv \mathbf{v}$. But $\mathbf{v}_{\text {field }} \neq$

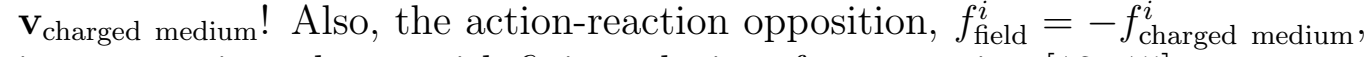
is not true in a theory with finite velocity of propagation [18, 15].

\subsection{What Are the Right Maxwell Equations of the Theory?}

Equation (25) is derived from Newton's second law of the theory, at least if the continuous medium behaves like a dust. Also, Assumption (ii) is necessary to the theory of gravitation. However, we may abandon Assumption (i), which means to write

$$
\boldsymbol{T}_{\text {total }}=\boldsymbol{T}_{\text {charged medium }}+\boldsymbol{T}_{\text {field }} \underline{+\boldsymbol{T}_{\text {interact }}}
$$

where $\boldsymbol{T}_{\text {interact }} \equiv \boldsymbol{T}_{\text {total }}-\boldsymbol{T}_{\text {charged medium }}-\boldsymbol{T}_{\text {field }}$ is some "interaction energy tensor". With (27), Assumption (ii) and Eq. (25) do not imply Eq. (26) any more, and do not determine the second group any more. One may postulate the standard gravitationally-modified second group (e.g. Refs. [14, 20]):

$$
F_{; \nu}^{\sigma \nu}=-\mu_{0} J^{\sigma}
$$

which, one may show [10], is writing almost the usual (3-vector-form) second group in terms of the local time and the space metric in the frame $\mathcal{E}$.

\section{Conclusion}

Maxwell equations for the "scalar ether theory" of gravity (SET) were proposed in a previous work [9]. They predict charge non-conservation in a variable gravitational field. This occurs already for a translation through SET's "ether". Using asymptotic PN and asymptotic "PM-like" expansions for the 
gravitational field and the e.m. field respectively, an explicit expression for the charge production rate $\hat{\rho}$ was obtained. For a group of Hertzian dipoles producing a strong but realistic e.m. field (and with a moderate translation velocity $V=10 \mathrm{~km} / \mathrm{s}),|\hat{\rho}|$ seems unrealistically high. In fact, those Maxwell equations are not consistent with the continuum dynamics of SET as applied to the e.m. field itself. There must be an additional, "interaction", energy tensor. Then the standard gravitationally-modified Maxwell equations become consistent with SET. The interaction energy might contribute to the "dark matter" [10].

\section{References}

[1] Arminjon M., A Theory of Gravity as a Pressure Force. I. Newtonian Space and Time, Rev. Roum. Sci. Techn. - Méc. Appl. 38 (1993) 3-21.

[2] Arminjon M., A Theory of Gravity as a Pressure Force. II. Lorentz Contraction and "Relativistic" Effects, Rev. Roum. Sci. Techn. - Méc. Appl. 38 (1993) 107128.

[3] Arminjon M., On the Extension of Newton's Second Law to Theories of Gravitation in Curved Space-Time, Arch. Mech. 48 (1996) 551-576.

[4] Arminjon M., Scalar Theory of Gravity as a Pressure Force, Rev. Roum. Sci. Tech.- Méc. Appl. 42 (1997) 27-57.

[5] Arminjon M., Accelerated Expansion as Predicted by an Ether Theory of Gravitation, Phys. Essays 14 (2001) 10-32.

[6] Arminjon M., Gravitational Effects on Light rays and Binary Pulsar Energy Loss in a Scalar Theory of Gravity, Theor. Math. Phys. 140 (2004) 1011-1027. (Teor. Mat. Fiz. 140 (2004) 139-159.)

[7] Arminjon M., Space Isotropy and Weak Equivalence Principle in a Scalar Theory of Gravity, Braz. J. Phys. 36 (2006) 177-189.

[8] Arminjon M., Summary of a Non-Uniqueness Problem of the Covariant Dirac Theory and of Two Solutions of It, In: Geometry, Integrability and Quantization XIV, I. Mladenov, A. Ludu and A. Yoshioka (Eds), Avangard Prima, Sofia 2013, pp. 48-60. 
[9] Arminjon M., Continuum Dynamics and the Electromagnetic Field in the Scalar Ether Theory of Gravitation, Open Physics 14 (2016) 395-409.

[10] Arminjon M., Charge Conservation in a Gravitational Field in the Scalar Ether Theory, Preprint HAL-01564537 (2017).

[11] Cattaneo C., General Relativity: Relative Standard Mass, Momentum, Energy and Gravitational Field in a General System of Reference, Nuovo Cim. 10 (1958) 318-337.

[12] Fock V., The Theory of Space, Time and Gravitation, 2nd English edition, Pergamon, Oxford 1964. (First Russian edition: Teoriya Prostranstva, Vremeni i Tyagoteniya, GITTL, Moscow 1955.)

[13] Jackson J., Classical Electrodynamics (3rd edn), Wiley, Hoboken (N.J.) 1998.

[14] Landau L. and Lifshitz E., The Classical Theory of Fields, 3rd English edition, Pergamon, Oxford 1971. (First Russian edition: Teoriya Polya, GITTL, Moscow and Leningrad 1941.)

[15] Mazilu P., Actio-Reactio Equations with Finite Wave Speed Derived from the Principle of Inertia, Acta Mech. 79 (1989) 233-257.

[16] McDonald K., Radiation in the Near Zone of a Hertzian Dipole, online document at www.physics.princeton.edu/ ${ }^{\sim}$ cdonald/examples/nearzone.pdf, 2004 .

[17] Møller C., The Theory of Relativity, Clarendon Press, Oxford 1952.

[18] Poincaré H., La Théorie de Lorentz et le Principe de Réaction, Arch. Néerland. Sci. Ex. Nat. (2) (1900) 252-278. Reprinted in Euvres Complètes, tome IX, Gauthier-Villars, Paris 1954, pp. 464-488.

[19] Prokhovnik S., The Logic of Special Relativity, Cambridge University Press, Cambridge (U.K.) 1967.

[20] Stephani H., General Relativity - an Introduction to the Theory of the Gravitational Field, Cambridge University Press, Cambridge (U.K.) 1982, p. 74. 